\title{
Visualisation of exercise-induced ischaemia of the right ventricle by thallium-201 single photon emission computed tomography
}

\author{
Junya Chiba, Yasuchika Takeishi, Shinya Abe, Hitonobu Tomoike
}

\begin{abstract}
Objective-Exercise thallium-201 (201 T1) single photon emission computed tomography (SPECT) has been used to detect potential ischaemia in the left ventricular myocardium but not in the right ventricle. The purpose of this study was to establish the clinical usefulness of a right ventricular polar map of ${ }^{201} \mathrm{~T} 1$ SPECT for visualisation of exercise-induced right ventricular ischaemia.

Methods-Myocardial ${ }^{201}$ T1 SPECT was obtained immediately after treadmill exercise in 97 patients with suspected coronary artery disease. A region of interest was placed over the right ventricle (RV) on post-stress transaxial images. Short axis images of this region were generated and reconstructed as a bull's eye polar map. Normal ranges of RV ${ }^{201} T 1$ uptake were determined in 12 patients with normal coronary arteries. Scintigraphic criteria for identifying RV perfusion abnormality were derived from 25 patients with right coronary artery (RCA) stenosis greater than $75 \%$. These criteria were applied to 60 consecutive patients with suspected coronary artery disease.
\end{abstract}

Results-Perfusion defects in the RV were larger in patients with proximal RCA stenosis than in those with distal RCA stenosis (mean (SD) 28 (16)\% v 6 (5)\%, $\mathrm{P}<$ $0.001)$. The sensitivity and specificity of the RV polar map for the detection of proximal RCA stenosis were $67 \%(8 / 12)$ and $98 \%$ (47/48), respectively. RV perfusion defects became undetectable in 9 patients who had successful percutaneous transluminal coronary angioplasty to a proximal RCA lesion.

Conclusions-A right ventricular polar map display was useful for visualising exercise-induced right ventricular ischaemia.

(Heart 1997;77:40-45) Yamagata University School of Medicine, Yamagata, Japan J Chiba Y Takeish S Abe

H Tomoike

Correspondence to: Dr H Tomoike, First Department of Internal Medicine, Yamagata University School of Medicine, 2-2-2 Iida-Nishi, Yamagata 990-23, Japan. Accepted for publication 22 May 1996
Exercise-induced right ventricular ischaemia is occasionally detected by currently available methods, such as an abnormal ejection fraction response to exercise or reduced regional wall motion on radionuclide ventriculography ${ }^{12}$ or on cross sectional echocardiography. ${ }^{3}$
Myocardial perfusion imaging with thallium$201\left({ }^{201} \mathrm{Tl}\right)$ has been accepted widely as a practical and sensitive tool for assessing acute or chronic coronary artery disease. Reduced perfusion was rarely detected in patients with functional abnormalities in the right ventricle. ${ }^{4}$ However, several reports have commented that perfusion defects in the right ventricle were detectable by exercise planar ${ }^{201} \mathrm{Tl}$ imaging in patients with right coronary artery disease. ${ }^{56}$ DePuey et $a l^{7}$ have also showed that myocardial single photon emission computed tomography (SPECT) with technetium-99m sestamibi but not ${ }^{201} \mathrm{Tl}$ detected abnormal distribution of right ventricular perfusion. The purpose of the present study was (a) to visualise ${ }^{201} \mathrm{Tl}$ uptake of the right ventricle on a bull's eye polar map, (b) to examine the sensitivity and specificity of the present image analysis of a exercise myocardial perfusion test, and (c) to assess the effect of percutaneous transluminal coronary angioplasty (PTCA) of the stenosed right coronary artery on right ventricular myocardial perfusion.

\section{Patients and methods}

SUBJECTS AND STUDY PROTOCOL

Ninety seven patients with suspected coronary artery disease who underwent both exercise myocardial ${ }^{201} \mathrm{Tl}$ SPECT and coronary arteriography were enrolled. None of them had previously undergone PTCA or coronary artery bypass grafting. The present study consisted of the following three protocols. In protocol I, normal files for ${ }^{201} \mathrm{Tl}$ distribution in the right ventricle were developed in 12 patients with normal coronary arteries (seven men and five women, mean age 62 years (range 42-68)). Then the normal files for the right ventricle were compared with the files of 25 patients (19 men and six women, mean age of 61 years (range 45-72)) who had either a proximal or a distal right coronary artery stenosis, and the criteria for identifying the presence of proximal right coronary artery stenosis were derived. Fifteen patients had a $75 \%$ or more stenosis of the proximal right coronary artery (defined as segment 1 or 2 in American Heart Association criteria), and 10 patients had a $75 \%$ or more stenosis of the distal right coronary artery (segment 3 or 4 ). In protocol II, these criteria were tested prospectively in 60 consecutive patients (44 men and 16 women, mean age 62 years (38-76)) with suspected coronary artery disease. Twelve had proximal right coronary artery stenosis and 48 did not. Then the diagnostic accuracy of the criteria 
A

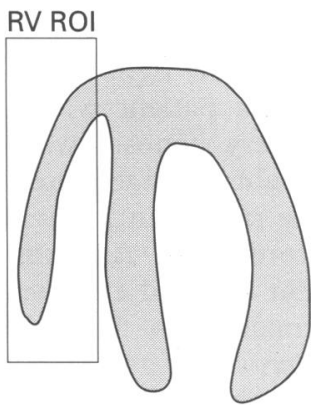

B

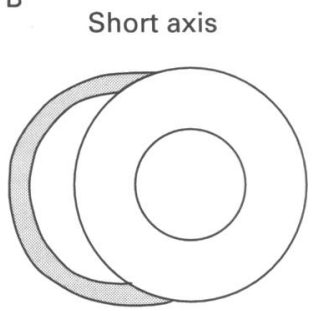

C

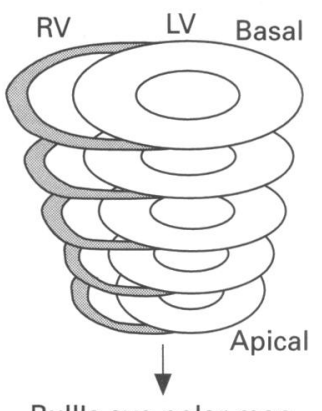

Bull's eye polar map

Figure 1 Reconstruction of right ventricular tomographic images. (A) Determination of ROI for the right ventricular free wall on the transaxial image. (B) Short axis tomographic images of the right ventricle. (C) $A$ display of bull's eye polar map for RV. Details in the text. $R V$, right ventricle; $R O I$, region of interest; $L V$, left ventricle. was tested. In protocol II, 14 patients had a previously documented myocardial infarction. One of them had evidence of right ventricular infarction. Significant coronary stenoses exceeding $50 \%$ in the left anterior descending artery and left circumflex artery were seen in 25 and 12 patients, respectively. Out of 97 patients in protocol I and II, nine patients underwent PTCA to the proximal portion of the right coronary artery. In protocol III, exercise ${ }^{201} \mathrm{Tl}$ scintigraphy was again performed within one week after the PTCA procedure, and changes in myocardial perfusion of the right ventricle were assessed before and after PTCA. All scintigrams were obtained for clinical purposes, and arteriography was performed for the diagnosis of coronary artery disease after the patient gave informed consent. The study protocol was approved by the Yamagata University Committee on Human Research.

\section{EXERCISE ${ }^{201}$ T1 IMAGING}

Exercise protocol

Antianginal medications were stopped at least 24 hours before the exercise tests, except for short-acting sublingual nitrates for episodes of angina. Exercise was carried out on a treadmill by means of a Bruce protocol. ${ }^{8}$ Blood pressure was measured every minute in the left arm by a standard cuff method, and an electrocardiogram was continuously monitored during the test. Exercise was terminated when angina, dyspnoea, ischaemic ST depression, serious arrhythmia, or fatigue occurred. A dose of 111 $\mathrm{MBq}$ of ${ }^{201} \mathrm{Tl}$ was injected rapidly 1 minute before the end of exercise.

\section{Data acquisitions}

Cardiac imaging started about five minutes after the ${ }^{201} \mathrm{Tl}$ injection. All studies were obtained on a rotating gamma camera (ZLC7500 Digitrac, Siemens) equipped with a parallel hole, high resolution collimator (iodine-123 $160 \mathrm{keV}$ Parallel, Nuclear Fields). Energy discrimination was provided by a $20 \%$ window centred at about $70 \mathrm{keV}$. Thirty two planar images were obtained over a 180 degree arc from the 45 degree right anterior oblique to the 45 degree left posterior oblique positions. ${ }^{9}$ Each image was accumulated for 40 seconds. The data were stored on a $64 \times 64$ matrix.

\section{Data processing}

Reconstruction of right ventricular images-Data processing was performed on a nuclear medicine computer system (Scintipac-700, Shimadzu). A series of contiguous transaxial images, $6 \mathrm{~mm}$ thick, were reconstructed by means of a filtered back projection algorithm without attenuation correction. ${ }^{9}$ To mask the left ventricle, a region of interest (ROI), which was horizontal to the interventricular septum, was placed over the right ventricle on the poststress transaxial image as shown in fig $1 \mathrm{~A}$. Transaxial right ventricular images within the ROI were further processed to obtain the short axis tomograms of the right ventricle (fig 1B) which were perpendicular to the long-axis of the ventricle.
Visualisation of right ventricle-A computer operator selected the right ventricular short axis slices from base to apex, approximately 6 to 8 slices in the normal sized heart (fig 1C). A short axis slice was divided into 30 sectors over 180 degrees, and the maximum counts per pixel within each sector were determined. Then these 30 values were normalised to maximal counts in the image and plotted as a circumferential profile of the normalised maximal counts versus angular location. ${ }^{10}$ Circumferential profile analysis was applied to each of the short axis slices from the base to the apex. These data were plotted in polar coordinates and arranged into a bull's eye polar map with the apex at the centre and the base toward the periphery.

Extent score of underperfusion in right ventricular images-When patients were studied each pixel was compared with the corresponding pixel in the normal profile. Pixels that were more than two standard deviations below the mean were defined as abnormal and displayed on a colour-coded standard deviation map. ${ }^{10}$ The ratio of the number of abnormal pixels to the total number of pixels was defined as an extent score.

\section{CORONARY ARTERIOGRAPHY}

Coronary arteriography was performed using the standard Judkins' technique. All angiograms were assessed by two experienced cardiologists who did not know any information regarding the patients. Significant coronary stenosis was defined as a luminal diameter narrowing of $75 \%$ or more in either the main coronary arteries or their major branches.

\section{STATISTICAL ANALYSIS}

The values of the data were reported as a mean (1SD). Extent score of ${ }^{201} \mathrm{Tl}$ defects was compared using Student's $t$ test. A P value $<0.05$ was considered significant.

\section{Results}

RECONSTRUCTION OF RIGHT VENTRICULAR

IMAGES ON A POLAR MAP

Protocol I

Right ventricular uptake was 28 (7)\% of that in the left ventricle in control subjects. Representative short axis images of the right ventricular free wall in a normal subject reconstructed as a bull's eye polar map are shown in fig 2. Right ventricular ${ }^{201} \mathrm{Tl}$ uptake was $33 \%$ of the left ventricular free wall in this case. Figure 3 shows polar maps of exercise ${ }^{201} \mathrm{Tl}$ SPECT of the left ventricle (upper panel) and of the right ventricle (lower panel) in a patient with right ventricular infarction. There was total occlusion of the proximal right coronary artery. The inferoposterior wall of the left ventricle was supplied by a posterior descending branch of the left circumflex artery. Although the perfusion defect was not detectable on the conventional left ventricular polar map (fig 3 upper panel), the right ventricular polar map clearly showed a large perfusion defect in the right ventricular free wall (fig 3 lower panel). 


\section{short axis images of the right ventricle} 19.
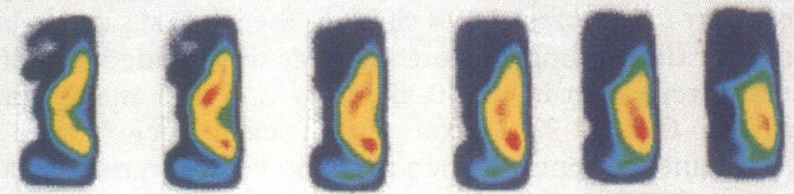

$\longleftarrow$ basal

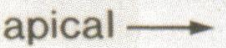

RV bull's eye map

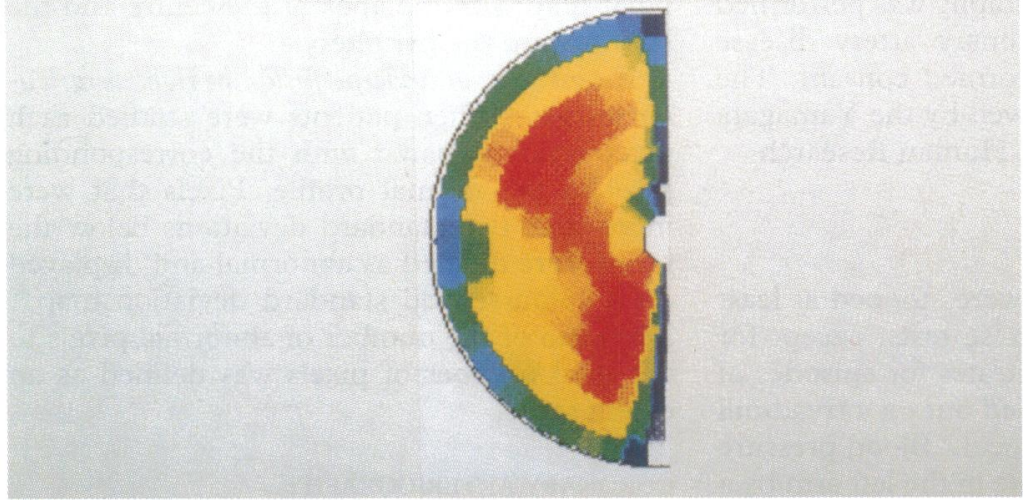

Figure 2 Short axis images and a polar map of the right ventricle in a 62-year-old woman with normal coronary arteries. Seven short axis planes derived from the transaxial frames are reconstructed as a bull's eye map (lower panel).
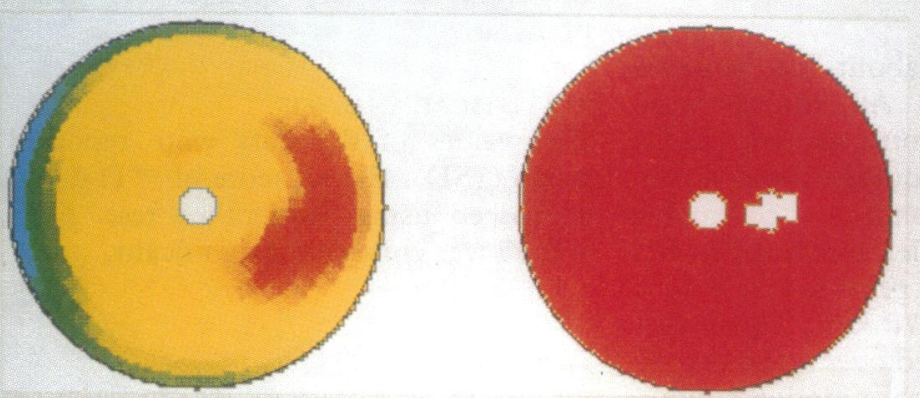

extent score $=2$
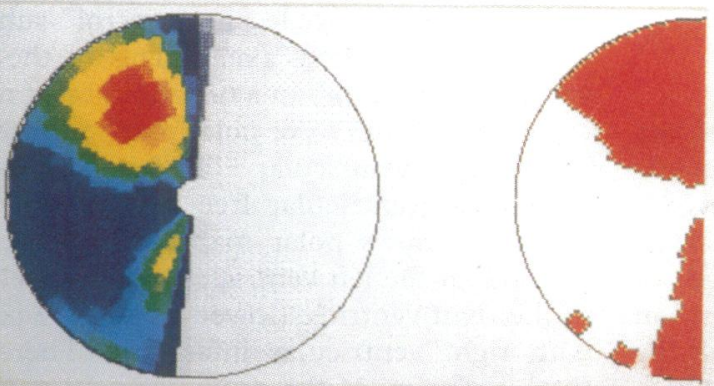

extent score $=56$

Figure 3 Polar map of a 62 year old man. The patient had the total occlusion of the proximal right coronary artery. The inferoposterior wall of the left ventricle was supplied by a posterior descending branch of the left circumflex artery. The upper panels show the conventional left ventricular polar map of ${ }^{201} \mathrm{Tl}$ uptake (left panel) and the extent score map (right panel). The lower panels are those reconstructed from the right ventricle. Decreased myocardial uptake of ${ }^{201} \mathrm{Tl}$ is evident at the middle to posterior portion in the right ventricle but not in the left ventricle.
Figure 4 shows the representative ${ }^{201} \mathrm{Tl}$ images of patients with a proximal (upper panel) and a distal (lower panel) right coronary artery stenosis. A large perfusion defect was demonstrable at the posterior wall of the right ventricle (extent score $=32$ ) in a patient with a proximal right coronary artery stenosis, while in a patient with a distal right coronary artery stenosis, ${ }^{201} \mathrm{Tl}$ uptake in the right ventricle was homogeneous (extent score $=2$ ). As summarised in fig 5 , the extent score of exerciseinduced perfusion abnormalities in the right ventricle was larger in patients with a proximal right coronary artery stenosis than in those with a distal right coronary artery stenosis (28 (16) $v 6(5), P<0.001)$. The extent scores of the RV were analysed twice at least two weeks apart to determine intraobserver variability in 10 patients. The calculation of RV extent score was highly reproducible in repeated measurements on separate occasions ( $r=$ $0.89, \mathrm{P}<0.01)$. When an extent score of more than 15 was chosen as a criterion of discriminating the presence or the absence of proximal right coronary artery stenosis, $10(67 \%)$ of 15 patients with a proximal right coronary stenosis were defined as abnormal, and all 10 patients with a distal right coronary stenosis were categorised as normal on perfusion images. Accordingly, we set a criterion of an extent score of greater than 15 for the later prospective study.

\section{APPLICATION OF RV POLAR MAP IN PROSPECTIVE STUDY \\ Protocol II}

The criterion derived from protocol I was applied prospectively to 60 consecutive patients. A proximal right coronary artery stenosis was found by angiography in 12 of 60 patients, and eight of these 12 patients showed significant right ventricular perfusion defects on the post-stress images (fig 6). An abnormal right ventricular ${ }^{201} \mathrm{Tl}$ defect was observed in only one of the 48 patients with no proximal right coronary stenosis. The sensitivity and specificity of the right ventricular polar map for detecting the presence of proximal right coronary artery stenosis were $67 \%$ and $98 \%$, respectively.

\section{CHANGES IN RIGHT VENTRICULAR PERFUSION AFTER PTCA \\ Protocol III}

PTCA of the proximal right coronary lesions was performed in nine of 12 patients with a proximal right coronary artery stenosis. All the angioplasties were angiographically successful. ${ }^{201}$ Tl SPECT was repeated within one week after PTCA. Figure 7 shows representative ${ }^{201}$ Tl RV polar maps and the extent scores before (upper panel) and after (lower panel) PTCA in a patient with effort angina. Before PTCA, an extensive ${ }^{201} \mathrm{Tl}$ defect was noted at the posterior portion of the polar map (extent score 40 ) that reflected the reduced right ventricular coronary blood flow during exercise caused by proximal right coronary artery stenosis. After the successful PTCA to the stenotic lesion, the right ventricular perfusion 


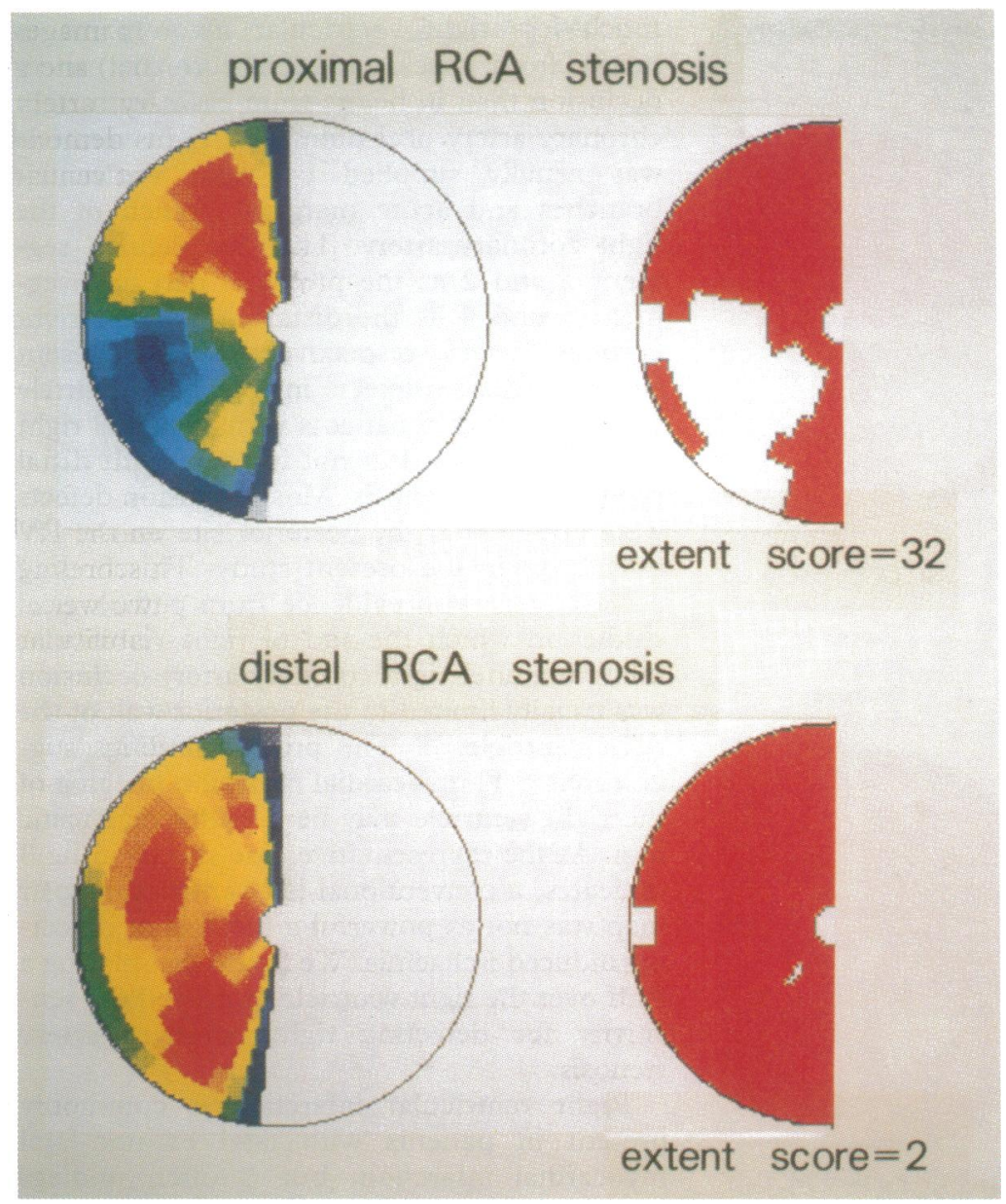

Figure 4 Upper panels show the right ventricular polar maps of a patient with the proximal right coronary stenosis. The left panel is a original polar map of ${ }^{201} \mathrm{Tl}$ uptake over the right ventricle, and the right panel is a standard deviation map of the left panel. Lower panels are ${ }^{201} \mathrm{Tl}$ uptake and its standard deviation map of a patient with distal right coronary artery stenosis. Right ventricular perfusion was reduced in the patient with proximal right coronary stenosis but not in the patient with distal right coronary artery stenosis.

Figure 5 Distribution of the right ventricular extent scores in patients with stenosis of the proximal or the distal right coronary artery in protocol I. Bar represents mean $(S D)$. $R P$, proximal right coronary artery stenosis; $R D$, distal right coronary artery stenosis. 0.01 ) (fig 8). defect disappeared. The extent score of right ventricular ${ }^{201} \mathrm{Tl}$ defects in the nine patients was consistently decreased to within normal limits after PTCA $(29(12) \% v 8(4) \%, \mathrm{P}<$

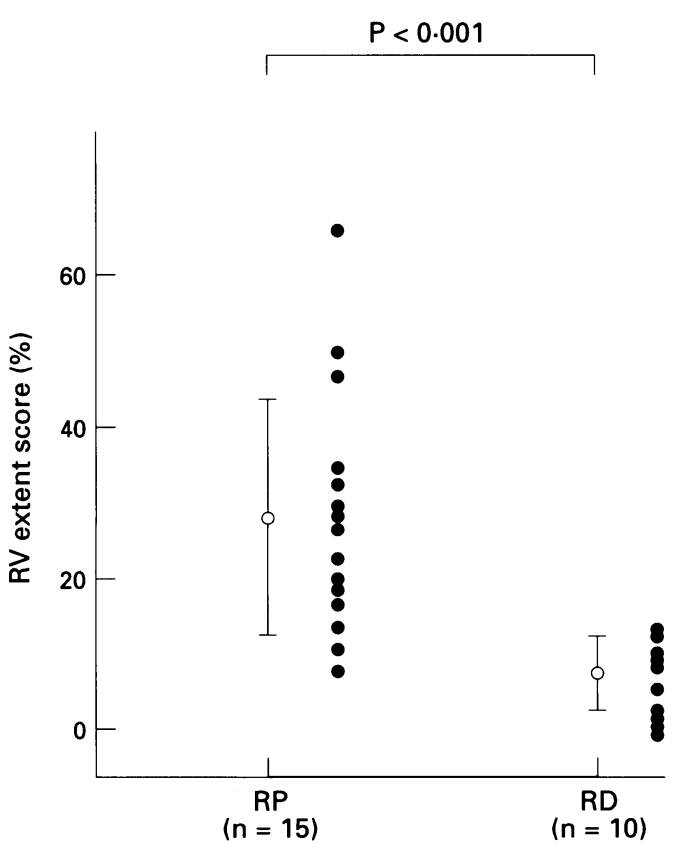

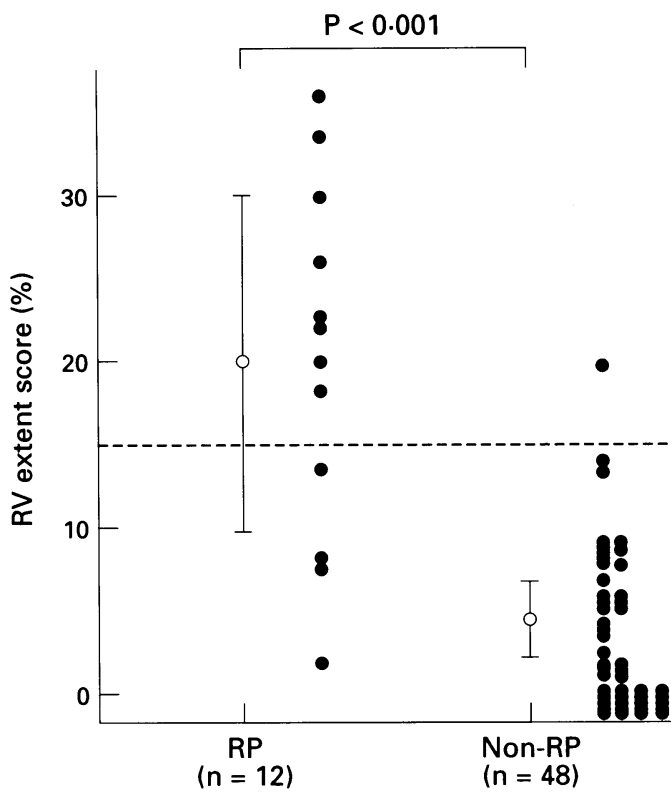

Figure 6 Distribution of the right ventricular extent scores in protocol II (the prospective study). The right ventricular extent score was significantly higher in patients with proximal stenosis of the right coronary artery than in those without. A dotted line represents the discrimination level of $15 \%$ for the extent score which was derived in the protocol I. RP, proximal right coronary artery stenosis.

\section{Discussion}

The results of this study indicate that exercise ${ }^{201} \mathrm{Tl}$ SPECT can be used to visualise exerciseinduced right ventricular ischaemia. By reconstructing right ventricular ${ }^{201} \mathrm{Tl}$ uptake during exercise as a polar map, we were able to measure the extent of induced ischaemia. We found that an extent score of greater than $15 \%$ in the right ventricular polar map was a practical criterion for determining the presence of stenosis of the proximal right coronary artery. Improvement in perfusion after successful PTCA of the right coronary artery extended the clinical applications of the RV polar map.The right ventricular free wall was not usually visible on resting ${ }^{201} \mathrm{Tl}$ images in normal cases. Exercise invariably increased the oxygen demand of the right ventricular myocardium as well as that of the left ventricle. This in turn increased myocardial blood flow and hence the amount of ${ }^{201} \mathrm{Tl}$ uptake in the myocardium. ${ }^{11} \mathrm{In}$ the present study, the right ventricle was well demonstrated on the exercise ${ }^{201} \mathrm{Tl}$ SPECT (fig 2). The ratio of myocardial ${ }^{201} \mathrm{Tl}$ activity in the right ventricle to the background was clearly increased on exercise. Thus the right ventricle can be imaged with ${ }^{201} \mathrm{Tl}$ on the exercise images but not on the rest or redistribution images. In the present study we did not show the data from redistribution or rest images. Because subjects in protocol II included only one patient with a right ventricular infarction, we consider that perfusion abnormalities on the exercise images indicate exercise-induced right ventricular ischaemia. In the present study, a high resolution collimator was used and relatively long acquisition time (40 seconds per projection) was needed to obtain enough counts in the right ventricle. Right ventricular thallium uptake was about 50 counts/pixel (about $25 \%$ of the left ventricle), 


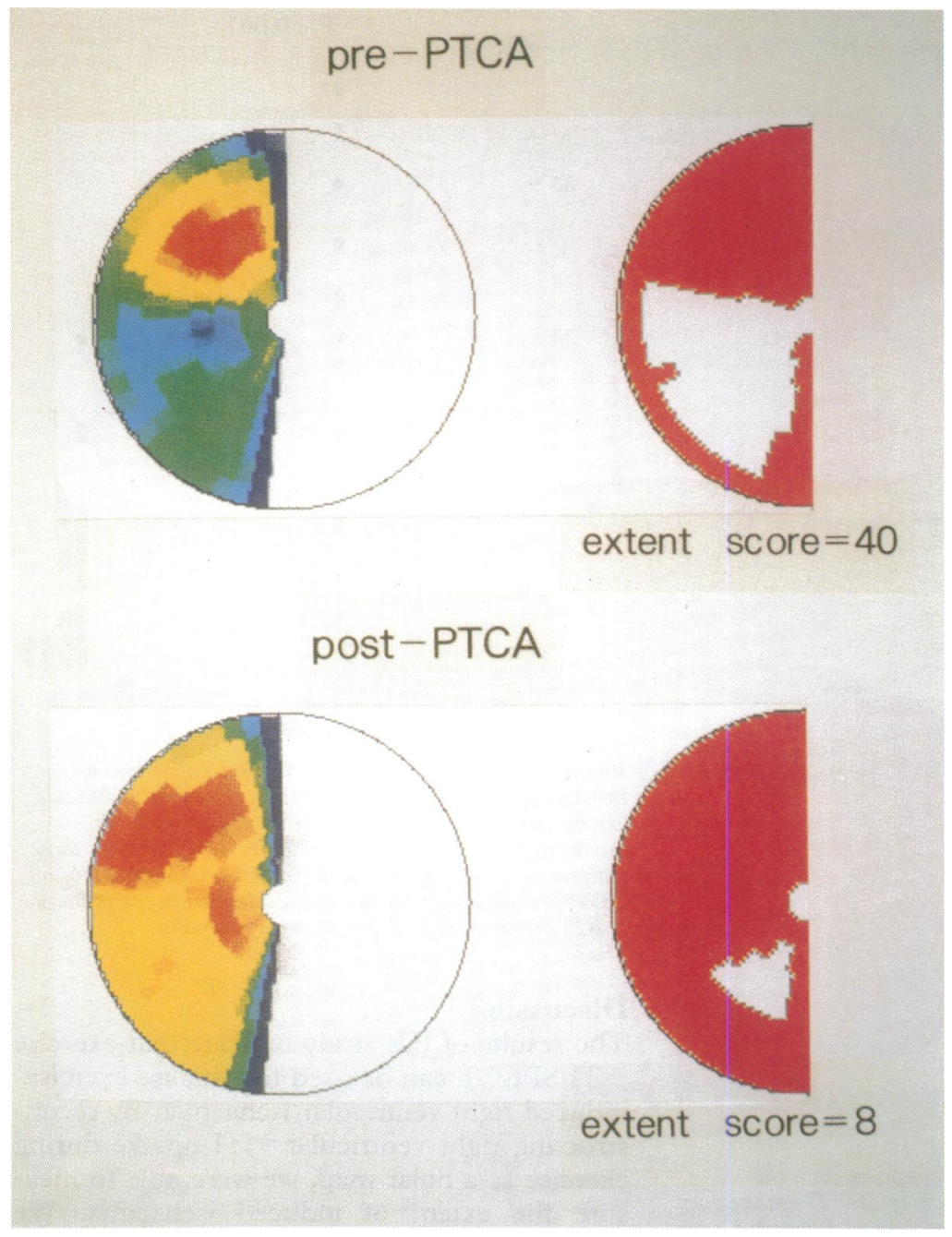

Figure 7 Representative right ventricular polar maps before and after PTCA in a 76 year old man with previous inferior myocardial infarction. This patient had had successful PTCA to the proximal right coronary artery. The size of a perfusion defect in the right ventricle before PTCA is $40 \%$ and the extent score was markedly reduced to $8 \%$ after successful revascularisation.

Figure 8 Plots show the extent scores of exerciseinduced perfusion abnormalities in the right ventricle before and afier PTCA. which was statistically enough for image analysis.

In an necropsy study Andersen et al ${ }^{12}$ found

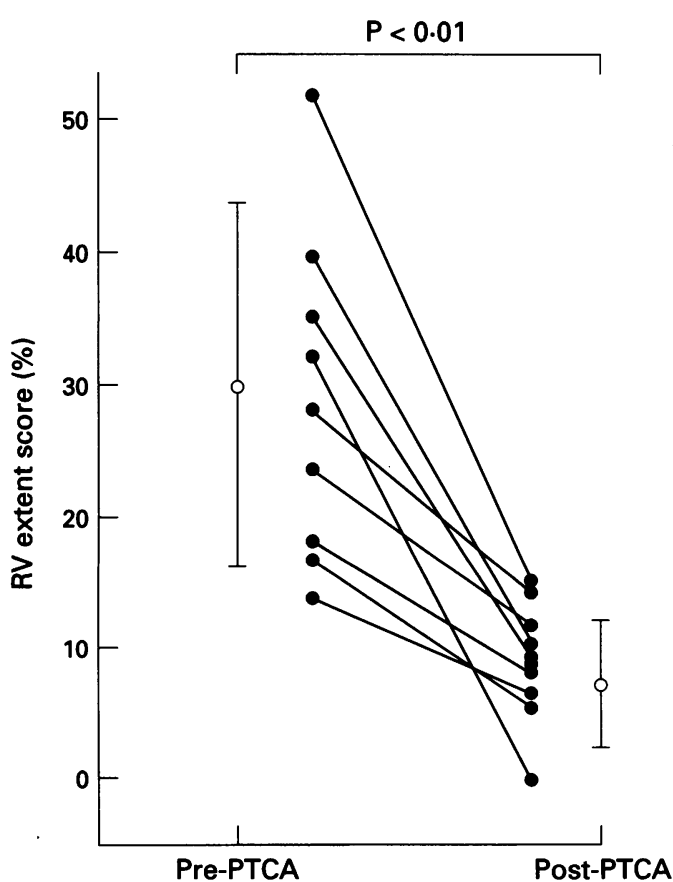

much less right ventricular involvement in hearts from cases with left coronary artery occlusion than in hearts from cases with right coronary artery occlusion. The right ventricle was usually supplied by right ventricular branches and acute marginal branch of the right coronary artery. Thus we defined segment 1 and 2 as the proximal part and segment 3 and 4 as the distal part of the right coronary artery, respectively. In the present study, perfusion defects in the right ventricle were identified in patients with proximal right coronary stenosis but not in those with distal right coronary stenosis. Most perfusion defects were observed at the posterior site on the RV polar map in the present study. This finding accords well with evidence from pathological studies in which the site of right ventricular infarction after right coronary artery occlusion was usually limited to the posterior wall of the right ventricle. ${ }^{12}{ }^{13}$ The present findings suggest that ${ }^{201} \mathrm{Tl}$ myocardial perfusion imaging of the right ventricle may be a useful diagnostic tool. As the representative case shown in fig 3 indicates, a conventional left ventricular polar map was not as powerful a method of detecting induced ischaemia. We found that placing a ROI over the right ventricle improved the sensitivity for detecting right coronary artery stenosis.

Right ventricular infarction is commonly present in patients with fatal or non-fatal myocardial infarction, but is often undiagnosed. ${ }^{13}$ Because right ventricular infarction is associated with an increased incidence of arrhythmia, systemic hypotension requiring volume loading, and/or right heart failure ${ }^{14}$ it is clinically important to identify such patients. Visual and quantitative assessment of right ventricular ${ }^{201} \mathrm{Tl}$ uptake as described in the present study may provide an important tool for choosing appropriate treatment.

We thank Dr A Komatani for discussion and $\mathrm{Mr}$ Kazue Takahashi for his technical assistance throughout the study. This study was supported in part by Grants-in-Aid for Scientific Research (Nos. 06454284, 07266202, 07557052, 08770488) from the Ministry of Education, Science and Culture, Japan.

1 Berger HJ, Johnstone DE, Sands JM, Gottschalk A, Zare BL. Response of right ventricular ejection fraction to upright bicycle exercise in coronary artery disease. Circulation 1979;60:1292-300.

2 Ratner SJ, Huang PJ, Friedman MI, Pierson RN. Assessment of right ventricular anatomy and function by Assessment of right ventricular anatomy and function by quantitative radionuclide

3 Lopez-Sendon J, Garcia-Fernandez MA, Coma-Canella I, Yanguela MM, Banuelos F. Segmental right ventricular function after acute myocardial infarction: two-dimensional echocardiographic study in 63 patients. $\mathrm{Am} \mathcal{F}$ Cardiol 1983;51:390-6.

4 Weiner BH, Alpert JS, Dalen JE, Ockene IS. Response of the right ventricle to exercise in patients with chronic heart disease. Am Heart $\mathcal{F}$ 1983;105:386-91.

5 Brachman MB, Rozanski A, Charuzi Y, Maddahi J, Waxman AD, Berman DS. Thallium-201 stress redistribution abnormalities of the right ventricle: A manifestation of proximal right coronary stenosis. Am Heart $¥ 1981$; 101:288-91.

6 Brown KA, Boucher CA, Okada RD, Strauss HW, Mckusick KA, Pohost GM. Serial right ventricular thallium-201 imaging after exercise: Relation to anatomy of lium-201 imaging after exercise: Relation to anatom

7 DePuey EG, Jones ME, Garcia EV. Evaluation of right ventricular regional perfusion with technetium-99mventricular regional perfusion with technetium-99m
Sestamibi SPECT. $₹$ Nucl Med 1991;32:1199-1205.

8 Takeishi Y, Tonooka I, Kubota I, Ikeda K, Masakane I, Chiba J, et al. Functional recovery of hibernating myocardium after coronary bypass surgery: Does it coincide with improvement in perfusion ? Am Heart $\mathcal{F} 1991$; 122:665-70. 
9 Takeishi Y, Tonooka I, Ikeda K, Komatani A, Tsuiki K, Yasui S. Dilatation of the left ventricular cavity on dipyridamole thallium-201 imaging: A new marker of triplevessel disease. Am Heart f 1991;121:466-75.

10 Takeishi Y, Tonooka I, Meguro M, Hoshi H, Masakane I, Ikeda $\mathrm{K}$, et al.The relationship between chest pain during thallium-201 scintigraphy with dipyridamole and myocardial ischaemia. Fpn Circ $\mathcal{f} 1991 ; 55: 465-72$

11 Parodi O, Neglia D, Marcassa C, Marzullo P, Sambuceti G. Right coronary artery disease. Pathophysiology, clinical relevance, and methods for recognition. Circulation 1991;
3:III-54-61.

12 Andersen HR, Falk E, Nielsen D. Right ventricular infarction: Frequency, size and topography in coronary heart disease: A prospective study comprising 107 consecutive autopsies from a coronary care unit. $\mathscr{F} \mathrm{Am}$ Coll Cardiol 1987;10:1223-32.

13 Isner JM, Roberts WC. Right ventricular infarction complicating left ventricular infarction secondary to coronary heart disease. Am $f$ Cardiol 1978;42:885-94.

14 Isner JM. Right ventricular myocardial infarction. $¥ A M A$ 1988;259:712-8.

\section{IMAGES IN CARDIOLOGY}

\section{A threatened paradoxical embolism}

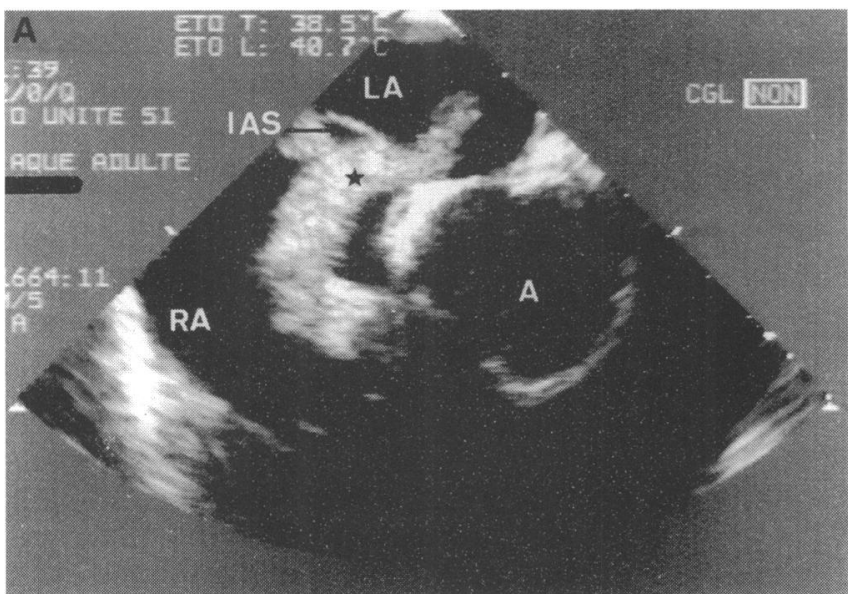

A 25 year old man with no medical history underwent splenectomy because of a post-traumatic haemorragic syndrome. Three weeks later he was admitted to hospital because of severe pulmonary embolism with haemodynamic instability. Transthoracic echocardiography detected a large thrombus in the right atrium. Phlebography identified a massive thrombosis in the left iliac artery. Open-heart surgery with full cardiopulmonary bypass was performed to remove the embolus from the inter-atrial septum as well as from the pulmonary artery. This embolectomy was guided by transoesophagal echocardiography which demonstrated direct evidence of an

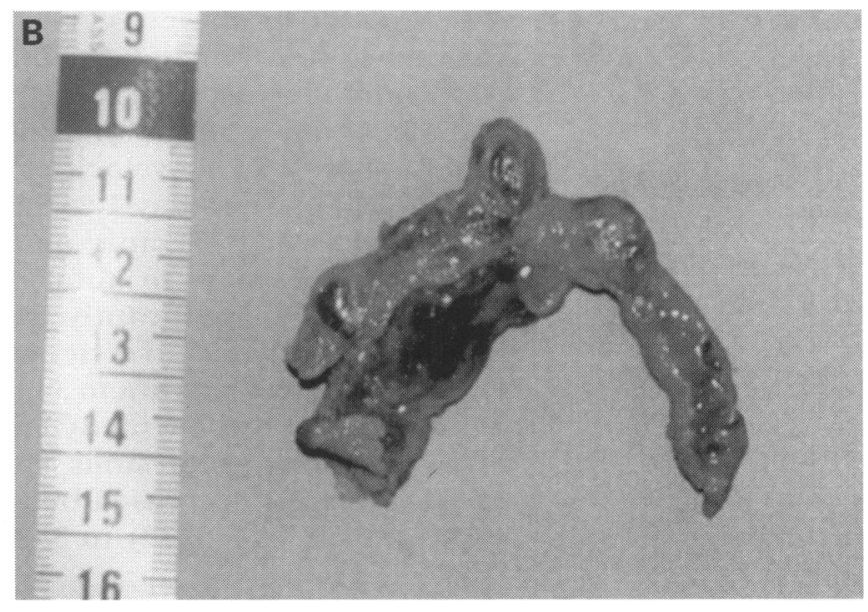

impending paradoxical embolism. A thrombus (black star in A) was seen trapped across the interatrial septum (IAS) and passing through a patent foramen ovale (PFO). Severe bowing of the IAS into the left atrium (LA) throughout the cardiac cycle indicated an increase in right atrial pressure. (RA, right atrium; A, aorta). A $10 \mathrm{~cm}$ thrombus was dislodged from the PFO (B). It is noteworthy that systemic embolism had not occurred. The patient remained well during three months of follow up.

PHIIIPPE CHEVALIER

JEAN-FRANCOIS OBADIA PAUL TOUBOUL 